\title{
First Direct Observation of Muon Antineutrino Disappearance
}

P. Adamson, ${ }^{7}$ C. Andreopoulos, ${ }^{20}$ D. J. Auty, ${ }^{24}$ D. S. Ayres, ${ }^{1}$ C. Backhouse, ${ }^{18}$ G. Barr, ${ }^{18}$ M. Bishai, ${ }^{3}$ A. Blake,,${ }^{5}$ G. J. Bock, ${ }^{7}$ D. J. Boehnlein, ${ }^{7}$ D. Bogert, ${ }^{7}$ S. Cavanaugh, ${ }^{9}$ D. Cherdack,${ }^{27}$ S. Childress, ${ }^{7}$ B. C. Choudhary, ${ }^{7}$ J. A. B. Coelho, ${ }^{6}$

S. J. Coleman, ${ }^{29}$ L. Corwin, ${ }^{12}$ D. Cronin-Hennessy, ${ }^{15}$ I. Z. Danko, ${ }^{19}$ J. K. de Jong, ${ }^{18}$ N. E. Devenish,${ }^{24}$ M. V. Diwan, ${ }^{3}$ M. Dorman, ${ }^{14}$ C. O. Escobar, ${ }^{6}$ J. J. Evans,${ }^{14}$ E. Falk, ${ }^{24}$ G. J. Feldman, ${ }^{9}$ M. V. Frohne, ${ }^{10}$ H. R. Gallagher, ${ }^{27}$ R. A. Gomes, ${ }^{8}$ M. C. Goodman, ${ }^{1}$ P. Gouffon, ${ }^{21}$ N. Graf,${ }^{11}$ R. Gran,${ }^{16}$ N. Grant,${ }^{20}$ K. Grzelak, ${ }^{28}$ A. Habig,,${ }^{16}$ D. Harris, ${ }^{7}$ J. Hartnell, ${ }^{24,20}$ R. Hatcher, ${ }^{7}$ A. Himmel,${ }^{4}$ A. Holin, ${ }^{14}$ C. Howcroft, ${ }^{4}$ X. Huang,,${ }^{1}$ J. Hylen, ${ }^{7}$ J. Ilic, ${ }^{20}$ G. M. Irwin, ${ }^{23}$ Z. Isvan, ${ }^{19}$ D. E. Jaffe, ${ }^{3}$ C. James, ${ }^{7}$ D. Jensen, ${ }^{7}$ T. Kafka, ${ }^{27}$ S. M. S. Kasahara, ${ }^{15}$ G. Koizumi, ${ }^{7}$ S. Kopp ${ }^{26}$ M. Kordosky, ${ }^{29}$ A. Kreymer, ${ }^{7}$ K. Lang, ${ }^{26}$ G. Lefeuvre, ${ }^{24}$ J. Ling, ${ }^{3,22}$ P. J. Litchfield, ${ }^{15,20}$ L. Loiacono, ${ }^{26}$ P. Lucas, ${ }^{7}$ W. A. Mann, ${ }^{27}$ M. L. Marshak, ${ }^{15}$ N. Mayer, ${ }^{12}$

A. M. McGowan, ${ }^{1}$ R. Mehdiyev, ${ }^{26}$ J.R. Meier,${ }^{15}$ M. D. Messier ${ }^{12}$ W. H. Miller, ${ }^{15}$ S. R. Mishra, ${ }^{22}$ J. Mitchell, ${ }^{5}$ C. D. Moore, ${ }^{7}$ J. Morfín, ${ }^{7}$ L. Mualem, ${ }^{4}$ S. Mufson, ${ }^{12}$ J. Musser, ${ }^{12}$ D. Naples,${ }^{19}$ J. K. Nelson, ${ }^{29}$ H. B. Newman,${ }^{4}$ R. J. Nichol, ${ }^{14}$ T. C. Nicholls, ${ }^{20}$ J. A. Nowak, ${ }^{15}$ J. P. Ochoa-Ricoux, ${ }^{4}$ W. P. Oliver, ${ }^{27}$ M. Orchanian, ${ }^{4}$ R. Ospanov, ${ }^{26}$ J. Paley, ${ }^{1,12}$ R. B. Patterson, ${ }^{4}$ G. Pawloski, ${ }^{23}$ G. F. Pearce,${ }^{20}$ D. A. Petyt, ${ }^{15}$ S. Phan-Budd, ${ }^{1}$ R. K. Plunkett, ${ }^{7}$ X. Qiu, ${ }^{23}$ J. Ratchford, ${ }^{26}$ T. M. Raufer, ${ }^{20}$ B. Rebel, ${ }^{7}$ P. A. Rodrigues, ${ }^{18}$ C. Rosenfeld, ${ }^{22}$ H. A. Rubin, ${ }^{11}$ M. C. Sanchez, ${ }^{13,1,9}$

J. Schneps,${ }^{27}$ P. Schreiner, ${ }^{1}$ P. Shanahan, ${ }^{7}$ A. Sousa, ${ }^{9}$ P. Stamoulis,${ }^{2}$ M. Strait,${ }^{15}$ N. Tagg, ${ }^{17}$ R. L. Talaga, ${ }^{1}$ E. Tetteh-Lartey, ${ }^{25}$ J. Thomas, ${ }^{14}$ M. A. Thomson, ${ }^{5}$ G. Tinti, ${ }^{18}$ R. Toner ${ }^{5}$ G. Tzanakos, ${ }^{2}$ J. Urheim, ${ }^{12}$ P. Vahle, ${ }^{29}$ B. Viren, ${ }^{3}$ A. Weber, ${ }^{18}$ R. C. Webb, ${ }^{25}$ C. White, ${ }^{11}$ L. Whitehead, ${ }^{3}$ S. G. Wojcicki, ${ }^{23}$ T. Yang, ${ }^{23}$ and R. Zwaska ${ }^{7}$

(MINOS Collaboration)

\author{
${ }^{1}$ Argonne National Laboratory, Argonne, Illinois 60439, USA \\ ${ }^{2}$ Department of Physics, University of Athens, GR-15771 Athens, Greece \\ ${ }^{3}$ Brookhaven National Laboratory, Upton, New York 11973, USA \\ ${ }^{4}$ Lauritsen Laboratory, California Institute of Technology, Pasadena, California 91125, USA \\ ${ }^{5}$ Cavendish Laboratory, University of Cambridge, Madingley Road, Cambridge CB3 OHE, United Kingdom \\ ${ }^{6}$ Universidade Estadual de Campinas, IFGW-UNICAMP, CP 6165, 13083-970, Campinas, SP, Brazil \\ ${ }^{7}$ Fermi National Accelerator Laboratory, Batavia, Illinois 60510, USA \\ ${ }^{8}$ Instituto de Física, Universidade Federal de Goiás, CP 131, 74001-970, Goiânia, GO, Brazil \\ ${ }^{9}$ Department of Physics, Harvard University, Cambridge, Massachusetts 02138, USA \\ ${ }^{10}$ Holy Cross College, Notre Dame, Indiana 46556, USA \\ ${ }^{11}$ Physics Division, Illinois Institute of Technology, Chicago, Illinois 60616, USA \\ ${ }^{12}$ Indiana University, Bloomington, Indiana 47405, USA \\ ${ }^{13}$ Department of Physics and Astronomy, Iowa State University, Ames, Iowa 50011, USA \\ ${ }^{14}$ Department of Physics and Astronomy, University College London, Gower Street, London WC1E 6BT, United Kingdom \\ ${ }^{15}$ University of Minnesota, Minneapolis, Minnesota 55455, USA \\ ${ }^{16}$ Department of Physics, University of Minnesota-Duluth, Duluth, Minnesota 55812, USA \\ ${ }^{17}$ Otterbein College, Westerville, Ohio 43081, USA \\ ${ }^{18}$ Subdepartment of Particle Physics, University of Oxford, Oxford OX1 3RH, United Kingdom \\ ${ }^{19}$ Department of Physics and Astronomy, University of Pittsburgh, Pittsburgh, Pennsylvania 15260, USA \\ ${ }^{20}$ Rutherford Appleton Laboratory, Science and Technologies Facilities Council, OX11 OQX, United Kingdom \\ ${ }^{21}$ Instituto de Física, Universidade de São Paulo, CP 66318, 05315-970, São Paulo, SP, Brazil \\ ${ }^{22}$ Department of Physics and Astronomy, University of South Carolina, Columbia, South Carolina 29208, USA \\ ${ }^{23}$ Department of Physics, Stanford University, Stanford, California 94305, USA \\ ${ }^{24}$ Department of Physics and Astronomy, University of Sussex, Falmer, Brighton BN1 9QH, United Kingdom \\ ${ }^{25}$ Physics Department, Texas A\&M University, College Station, Texas 77843, USA \\ ${ }^{26}$ Department of Physics, University of Texas at Austin, 1 University Station C1600, Austin, Texas 78712, USA \\ ${ }^{27}$ Physics Department, Tufts University, Medford, Massachusetts 02155, USA \\ ${ }^{28}$ Department of Physics, Warsaw University, Hoża 69, PL-00-681 Warsaw, Poland \\ ${ }^{29}$ Department of Physics, College of William \& Mary, Williamsburg, Virginia 23187, USA
}

(Received 2 April 2011; published 5 July 2011)

This Letter reports the first direct observation of muon antineutrino disappearance. The MINOS experiment has taken data with an accelerator beam optimized for $\bar{\nu}_{\mu}$ production, accumulating an exposure of $1.71 \times 10^{20}$ protons on target. In the Far Detector, 97 charged current $\bar{\nu}_{\mu}$ events are observed. The no-oscillation hypothesis predicts 156 events and is excluded at $6.3 \sigma$. The best fit to oscillation yields 
$\left|\Delta \bar{m}^{2}\right|=\left[3.36_{-0.40}^{+0.46}\right.$ (stat) \pm 0.06 (syst) $] \times 10^{-3} \mathrm{eV}^{2}, \sin ^{2}(2 \bar{\theta})=0.86_{-0.12}^{+0.11}$ (stat) \pm 0.01 (syst). The MINOS $\nu_{\mu}$ and $\bar{\nu}_{\mu}$ measurements are consistent at the $2.0 \%$ confidence level, assuming identical underlying oscillation parameters.

Observations by many experiments provide compelling evidence for neutrino oscillation [1-9]. This oscillation, a consequence of the quantum mechanical mixing of the neutrino mass and weak flavor eigenstates, is governed by the elements of the Pontecorvo-Maki-NakagawaSakata matrix [10], parameterized by three mixing angles and a $C P$ phase, and by two independent neutrino masssquared differences. As the measurement precision on oscillation parameters improves, so does the potential for observing new phenomena. In particular, measured differences between the neutrino and antineutrino oscillation parameters would indicate new physics. CPT symmetry, one of the most fundamental assumptions underlying the standard model, constrains the allowed differences in the properties of a particle and its antiparticle [11] and requires that their masses be identical. This symmetry has been extensively tested in other sectors, most notably the kaon sector [12]. Additionally, neutrinos passing through matter could experience nonstandard interactions [13] that alter the $\nu_{\mu}$ and $\bar{\nu}_{\mu}$ disappearance probabilities and, thus, the inferred oscillation parameters [14].

The MINOS experiment has used a $\nu_{\mu}$ beam to measure the larger (atmospheric) mass-squared difference $\left|\Delta m^{2}\right|=$ $\left(2.32_{-0.08}^{+0.12}\right) \times 10^{-3} \mathrm{eV}^{2}$ and the mixing angle $\sin ^{2}(2 \theta)>$ 0.90 (90\% confidence limit [C.L.]) through observation of $\nu_{\mu}$ disappearance $[3,15]$. The corresponding antineutrino oscillation parameters are much less precisely known.

This Letter describes the first direct observation of $\bar{\nu}_{\mu}$ disappearance consistent with oscillation, yielding the most precise measurement to date of the larger antineutrino mass-squared difference. The only previous measurements from $\bar{\nu}_{\mu}$-tagged samples, providing weak constraints, come from the MINOS atmospheric neutrino sample [16] and an analysis of the $\bar{\nu}_{\mu}$ component of the MINOS $\nu_{\mu}$ data sample $[17,18]$. The strongest indirect constraints come from a global fit [19], dominated by SuperKamiokande data which measure the sum of atmospheric $\nu_{\mu}$ and $\bar{\nu}_{\mu}$ interaction rates.

For this measurement the NuMI beam line [20] was configured to produce a $\bar{\nu}_{\mu}$-enhanced beam. The current in the magnetic horns was configured to focus negative pions and kaons produced by $120 \mathrm{GeV}$ protons incident on a graphite target. Most mesons travel along a $675 \mathrm{~m}$ long decay pipe, filled with helium at 0.9 atm, and decay to produce a $\bar{\nu}_{\mu}$-enhanced beam with a peak energy of $3 \mathrm{GeV}$ (see Fig. 1). Interactions of $\nu_{\mu}$ comprise a fraction of all charged current (CC) events in the MINOS detectors which rises from about $21 \%$ below $6 \mathrm{GeV}$ up to about $81 \%$ at
$20 \mathrm{GeV}$, in the case of no oscillation. The data set in this Letter corresponds to an exposure of $1.71 \times 10^{20}$ protons on target (POT).

The MINOS experiment uses two similar detectors located 1.04 [Near Detector (ND)] and $735 \mathrm{~km}$ [Far Detector (FD)] from the NuMI target. The $\bar{\nu}_{\mu} \mathrm{CC}$ interaction rate as a function of reconstructed $\bar{\nu}_{\mu}$ energy is measured in each detector. The measured FD energy spectrum is compared to that predicted by using the ND data. In this comparison, many sources of systematic uncertainty cancel. Antineutrino oscillation causes a deficit in the FD with an energy dependence, in the approximation of two-flavor mixing, of

$$
P\left(\bar{\nu}_{\mu} \rightarrow \bar{\nu}_{\mu}\right)=1-\sin ^{2}(2 \bar{\theta}) \sin ^{2}\left(\frac{1.267 \Delta \bar{m}^{2} L}{E}\right),
$$

where $L[\mathrm{~km}]$ is the distance from the point of antineutrino production, $E[\mathrm{GeV}]$ the $\bar{\nu}_{\mu}$ energy, $\Delta \bar{m}^{2}\left[\mathrm{eV}^{2}\right]$ the antineutrino mass-squared difference, and $\bar{\theta}$ the antineutrino mixing angle.

The MINOS detectors [21] are tracking calorimeters, formed of planes of steel interleaved with planes of scintillator. The scintillator is divided into strips with a width of $4.1 \mathrm{~cm}$. In CC interactions, $\bar{\nu}_{\mu}\left(\nu_{\mu}\right)+N \rightarrow \mu^{+}\left(\mu^{-}\right)+X$,

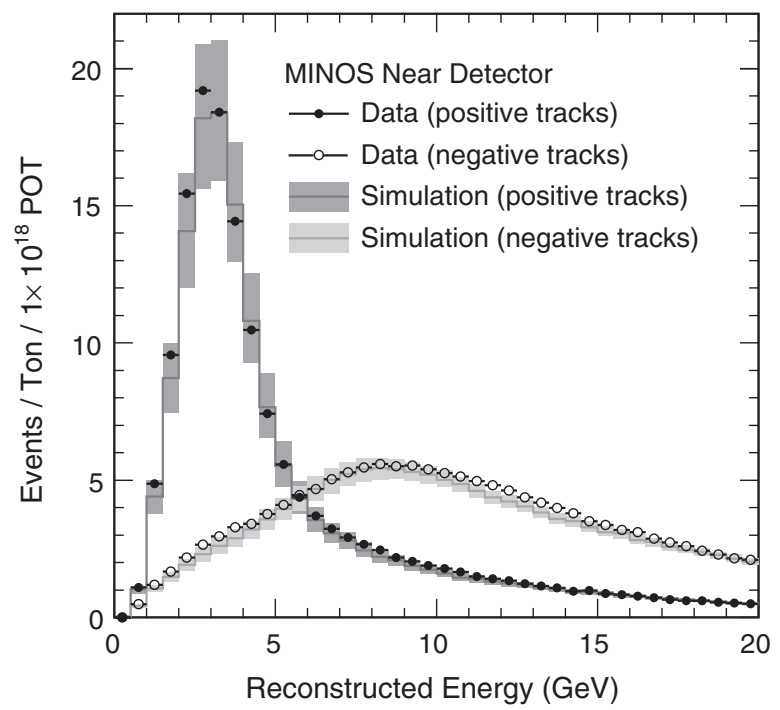

FIG. 1. The reconstructed energy spectra of events in the Near Detector classified as charged current interactions, separated according to the reconstructed charge of the track. The events with a negatively charged track are not used in the oscillation analysis. The shaded bands represent the systematic uncertainty on the simulation. 
a hadronic shower $(X)$ and a muon track may be observed. The hadronic energy is measured by summing the amount of light produced in the scintillator. Muon energy is measured by the range for contained tracks or, for exiting tracks, by the curvature in a $\sim 1.4 \mathrm{~T}$ toroidal magnetic field. The incoming neutrino energy is reconstructed as the sum of the hadronic and muon energies. For the data presented in this Letter, the fields in both detectors focus $\mu^{+}$and defocus $\mu^{-}$, allowing the separation of $\bar{\nu}_{\mu}$ and $\nu_{\mu}$ $\mathrm{CC}$ interactions on an event-by-event basis.

A sample of $\bar{\nu}_{\mu}$ CC interactions is isolated by identifying the presence of a positively charged track. Neutral current (NC) interactions produce only a hadronic shower at the vertex. Similarly, CC interactions of $\nu_{e}$ and $\bar{\nu}_{e}$ (which correspond to $2.0 \%$ of all CC interactions at the ND) produce only showerlike activity. The main background arises from tracks reconstructed out of shower activity. This background is reduced $[2,22]$ by a method which uses four variables to identify the presence of an isolated track with muonlike energy deposition. These four variables are the track length, the average pulse height per plane along the track, the transverse energy deposition profile of the track, and the fluctuation of the energy deposited in scintillator strips along the track, and are combined by using a $k$-nearest-neighbor $(k \mathrm{NN})$ algorithm [23] to produce a single output variable. The position of the selection cut on this variable is tuned to optimize the statistical sensitivity to $\left|\Delta \bar{m}^{2}\right|$, yielding the same selection criterion as for the MINOS $\nu_{\mu}$ analysis [2].

The charge of reconstructed muon tracks is determined by analyzing the curvature of the track in the magnetic field [24]. Figure 1 shows the reconstructed energy of selected $\mathrm{CC}$ events in the ND, separated according to the measured track charge sign. The events reconstructed with a negatively charged track consist primarily of $\nu_{\mu}$ CC interactions and are removed from further analysis. Events with a positively charged track form the selected $\bar{\nu}_{\mu} \mathrm{CC}$ sample and are used to predict the expected energy spectrum at the FD. Below $6 \mathrm{GeV}$, where the majority of the oscillation signal is expected, the selected $\bar{\nu}_{\mu} \mathrm{CC}$ sample at the ND has a purity, obtained from the simulation, of $98 \%$ (the background consisting of $1 \% \mathrm{NC}$ events and $1 \% \nu_{\mu} \mathrm{CC}$ events). Above $6 \mathrm{GeV}$, the purity is $88 \%$, and the contamination is primarily $\nu_{\mu}$ CC events; higher momentum muons follow a less curved path, giving a greater probability of charge misidentification. The total $\bar{\nu}_{\mu} \mathrm{CC}$ reconstruction and selection efficiency is $93 \%$.

The measured ND energy spectrum is used to predict the FD spectrum, as previously $[1,2,17]$. This procedure is particularly effective in mitigating sources of systematic uncertainty which affect both detectors similarly. For example, uncertainties on the neutrino flux and cross sections dominate the systematic error band on the ND energy spectrum, shown in Fig. 1, but have a negligible impact on the oscillation measurement.
The production of hadrons in the NuMI target is constrained by fits to the ND data [1,2]. These fits use data from the $\nu_{\mu}$ beam to determine the $\pi$ and $K$ yields as a function of their transverse and longitudinal momenta at production. Recent measurements [25] of the ratio of $\pi^{+} / \pi^{-}$yields are included as constraints in these fits. This tuning procedure improves agreement between the simulated ND energy spectrum and the data but does not significantly affect the predicted FD energy spectrum. Uncertainties on the modeling of the beam have a negligible effect on the predicted FD energy spectrum and are accounted for in the oscillation measurement.

The same event selection criteria are used in both detectors. The FD data selection was determined by using simulation and ND data, before the FD data was examined. All FD events passing the $k \mathrm{NN}$ selection are shown in Fig. 2, distributed according to the sign of the reconstructed track charge, divided by the momentum. The figure shows good modeling of track charge identification. Events with a negatively charged track are minimally affected by oscillation due to their higher mean energy and are removed from further analysis.

The systematic uncertainty on the oscillation parameters is much smaller than the statistical uncertainty. The sources of systematic uncertainty are very similar to those discussed for the MINOS $\nu_{\mu}$ analysis [3]. An additional uncertainty is estimated on the level of $\nu_{\mu}$ CC background in the selected $\bar{\nu}_{\mu} \mathrm{CC}$ event sample; below $6 \mathrm{GeV}$, the purity of the selected $\bar{\nu}_{\mu}$ CC sample is known to within $1 \%$. To evaluate this uncertainty, the simulated background is scaled until the total number of simulated and data events matches in the background-enhanced set of events which fail the $k \mathrm{NN}$ selection criterion. This scale factor is

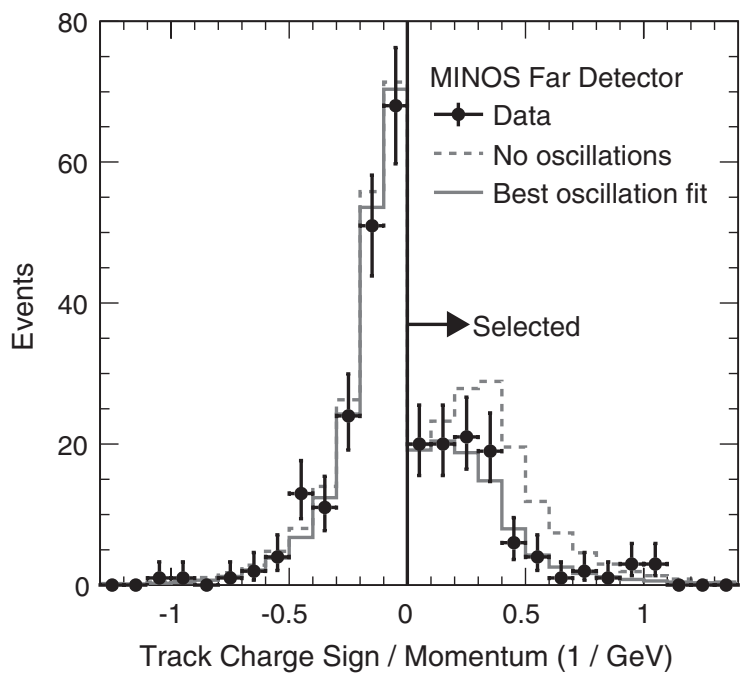

FIG. 2. The distribution of the sign of the reconstructed charge divided by the momentum of selected muon tracks in the Far Detector. The simulated distribution is shown in the case of no oscillation and oscillation assuming the best-fit $\nu_{\mu}$ parameters from Ref. [3] and $\bar{\nu}_{\mu}$ parameters from this analysis. 
taken as the uncertainty on the level of background in the selected $\bar{\nu}_{\mu}$ CC sample. The total systematic uncertainty on the measurement of $\left|\Delta \bar{m}^{2}\right|$ is $+0.063-0.060 \times$ $10^{-3} \mathrm{eV}^{2}$; on the measurement of $\sin ^{2}(2 \bar{\theta})$, the total systematic uncertainty is \pm 0.012 .

By using the prediction obtained from the ND data, 156 selected $\bar{\nu}_{\mu} \mathrm{CC}$ events with energy below $50 \mathrm{GeV}$ are expected in the FD in the absence of oscillation while 97 events are observed. The energy spectra of these FD events are shown in Fig. 3. A clear energy-dependent deficit is observed, showing the first conclusive evidence for $\bar{\nu}_{\mu}$ disappearance consistent with oscillation in a $\bar{\nu}_{\mu}$-tagged sample. The no-oscillation hypothesis is disfavored at 6.3 standard deviations.

Oscillation is incorporated into the predicted energy spectrum according to Eq. (1). By comparing the prediction to the data using a binned log likelihood, oscillation parameters are found which maximize the likelihood. These are $\left|\Delta \bar{m}^{2}\right|=\left[3.36_{-0.40}^{+0.46}\right.$ (stat) $\pm 0.06($ syst $\left.)\right] \times$ $10^{-3} \mathrm{eV}^{2}$ and $\sin ^{2}(2 \bar{\theta})=0.86_{-0.12}^{+0.11}$ (stat) \pm 0.01 (syst) and are consistent with all previous direct limits [16-18]. The prediction for oscillation with these best-fit values is shown in Fig. 3 and includes $2 \mathrm{NC}$ events, $5 \nu_{\mu} \mathrm{CC}$ events, and 0.3 $\bar{\nu}_{\tau}$ CC events.

The confidence limits on the oscillation parameters, shown in Fig. 4, are calculated by using the FeldmanCousins technique [26], in which the effect of all sources of systematic uncertainty is included [27,28]. Figure 4 compares these limits to the previous best limit [19].

MINOS has measured the $\nu_{\mu}$ oscillation parameters to obtain a best fit of $\left|\Delta m^{2}\right|=2.32 \times 10^{-3} \mathrm{eV}^{2}$, $\sin ^{2}(2 \theta)=1.0$ [3]. Assuming that muon antineutrinos oscillate with these parameters, 110 selected events are

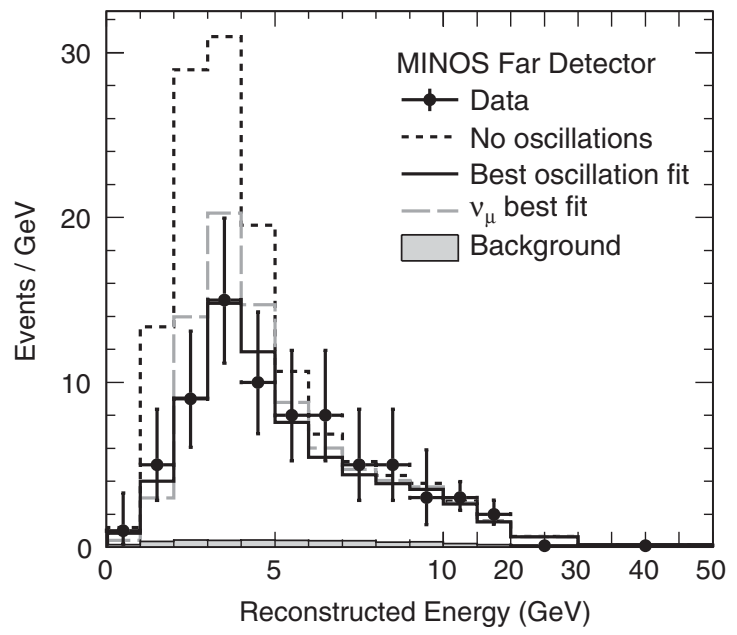

FIG. 3. Comparison of the measured Far Detector $\bar{\nu}_{\mu} \mathrm{CC}$ energy spectrum to the expectation in three cases: in the absence of oscillation, using the oscillation parameters which best fit these $\bar{\nu}_{\mu}$ data (for this case, the total expected background is also indicated), and using the best-fit $\nu_{\mu}$ oscillation parameters measured by MINOS [3]. expected in the FD below $50 \mathrm{GeV}$. This expected energy spectrum is shown in Fig. 3, denoted as " $\nu_{\mu}$ best fit."

Figure 4 compares the MINOS measurements of the $\nu_{\mu}$ and $\bar{\nu}_{\mu}$ oscillation parameters. In both measurements, the purity of the event samples in the oscillation region is high. Below $6 \mathrm{GeV}$, there is no more than $3 \% \nu_{\mu} \mathrm{CC}$ contamination in the $\bar{\nu}_{\mu}$ CC sample and vice versa. Therefore the measurements of the $\nu_{\mu}$ and $\bar{\nu}_{\mu}$ oscillation parameters are nearly independent. Since the $\bar{\nu}_{\mu}$ measurement is heavily statistically limited, the impact of correlated systematic uncertainties is negligible.

In a joint fit to the data used in the MINOS $\nu_{\mu}$ and $\bar{\nu}_{\mu}$ measurements, assuming identical $\nu_{\mu}$ and $\bar{\nu}_{\mu}$ oscillation parameters, the best-fit parameters are $\left|\Delta \bar{m}^{2}\right| \equiv\left|\Delta m^{2}\right|=$ $2.41 \times 10^{-3} \mathrm{eV}^{2}, \sin ^{2}(2 \bar{\theta}) \equiv \sin ^{2}(2 \theta)=0.97$. The significance of the difference in likelihood between this best fit and the fits to the individual $\nu_{\mu}$ and $\bar{\nu}_{\mu}$ data sets is evaluated by using a Feldman-Cousins approach [27]. Ten thousand simulated experiments are generated, assuming the joint best-fit oscillation parameters above, and include all sources of systematic uncertainty. The difference in likelihood between the joint and individual $\nu_{\mu}$ and $\bar{\nu}_{\mu}$ fits is recorded for each experiment, and the fraction of simulated experiments with a difference in likelihood larger than that observed in the data is a measure of the significance of the observed difference. Assuming identical $\nu_{\mu}$ and $\bar{\nu}_{\mu}$ oscillation parameters, the probability that the MINOS measurements of the $\nu_{\mu}$ and $\bar{\nu}_{\mu}$ parameters would be more discrepant than those observed is $2.0 \%$.

A thorough search for sources of mismodeling that could provide a difference between the $\nu_{\mu}$ and $\bar{\nu}_{\mu}$ measurements was performed. The only change between $\nu_{\mu}$ and $\bar{\nu}_{\mu}$

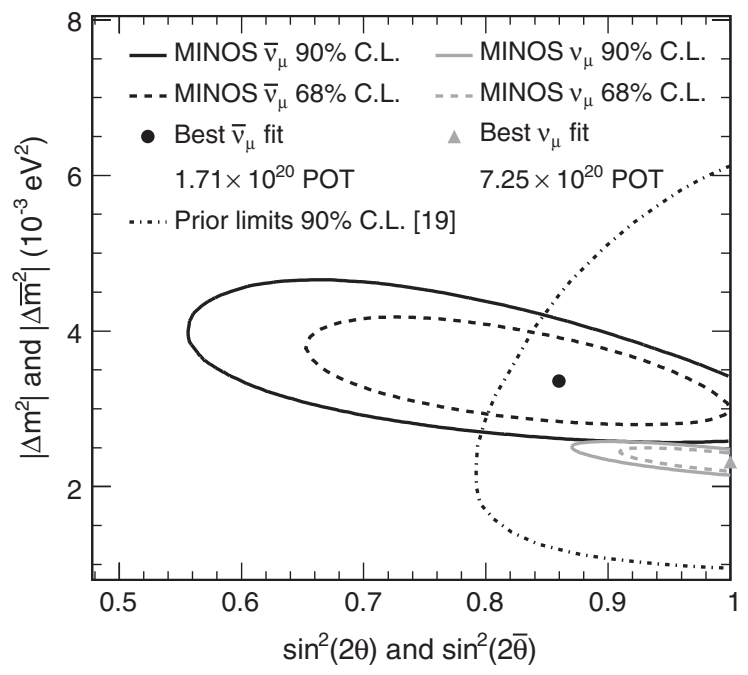

FIG. 4. Allowed regions for the $\bar{\nu}_{\mu}$ oscillation parameters from a fit to the data in Fig. 3, including all sources of systematic uncertainty. Indirect limits prior to this work [19] and the MINOS allowed region for $\nu_{\mu}$ oscillation [3] are also shown. 
running modes was the reversal of the directions of the current in the focusing horns of the beam and of the magnetic fields in the detectors. Very similar data analysis procedures are used in both modes, with the same reconstruction code and similar selection criteria used to obtain the event samples and the same technique used to obtain the FD predictions. These similarities make the comparison of $\nu_{\mu}$ and $\bar{\nu}_{\mu}$ measurements robust and limit the possible sources which could generate a spurious difference.

The $\nu_{\mu}$ and $\bar{\nu}_{\mu}$ analyses differ in that the $\bar{\nu}_{\mu}$-enhanced beam contains a significant $\nu_{\mu}$ component (which is effectively removed by the selection cuts). Figure 1 shows that this component is at high energy, away from the oscillation signal region, and therefore any residual contamination has little effect on the oscillation measurement. Figures 1 and 2 show the $\nu_{\mu}$ CC component to be well modeled in both detectors in both shape and normalization. All FD events were scanned by eye to ensure that the selection does not anomalously misclassify events by the sign of the charge. Performing the analysis without any selection on track charge in the FD produces consistent results. A scan by eye of events in the ND showed the track reconstruction efficiency to be well modeled.

Differences in the underlying inelasticity distributions for $\nu_{\mu}$ and $\bar{\nu}_{\mu}$ events lead to differences in the muon and hadron energy distributions for the two samples. Studies to validate the reconstruction of muon tracks and hadronic showers included the tightening of reconstruction quality criteria, investigation of the comparative performance of various detector regions, and the use of an alternative hadronic energy estimator. These studies show the detectors to be well modeled and that any mismodeling in reconstruction and selection efficiencies is accounted for in the systematic uncertainty. In conclusion, no evidence is found for any systematic error that could cause a significant difference between the measured $\nu_{\mu}$ and $\bar{\nu}_{\mu}$ oscillation parameters.

In summary, MINOS has used a beam optimized for the production of $\bar{\nu}_{\mu}$ to make the first direct observation of $\bar{\nu}_{\mu}$ disappearance and, assuming the disappearance is caused by oscillation, has made the most precise measurement of the corresponding antineutrino mass-squared difference to date. From fits to these data, MINOS has measured the oscillation parameters to be $\left|\Delta \bar{m}^{2}\right|=\left[3.36_{-0.40}^{+0.46}\right.$ (stat) \pm 0.06 (syst) $] \times 10^{-3} \mathrm{eV}^{2}$ and $\sin ^{2}(2 \bar{\theta})=0.86_{-0.12}^{+0.11}$ (stat) \pm 0.01 (syst). The MINOS $\nu_{\mu}$ and $\bar{\nu}_{\mu}$ measurements are consistent at the $2.0 \%$ confidence level, assuming identical underlying oscillation parameters. Additional data are currently being taken with the $\bar{\nu}_{\mu}$-enhanced NuMI beam, with the aim of doubling the statistics presented in this Letter.

This work was supported by the U.S. DOE; the United Kingdom STFC; the U.S. NSF; the State and University of Minnesota; the University of Athens, Greece; and Brazil's FAPESP and CNPq. We are grateful to the Minnesota Department of Natural Resources, the crew of the
Soudan Underground Laboratory, and the personnel of Fermilab for their contributions to this effort.

[1] D. G. Michael et al., Phys. Rev. Lett. 97, 191801 (2006); P. Adamson et al., Phys. Rev. D 77, 072002 (2008).

[2] P. Adamson et al., Phys. Rev. Lett. 101, 131802 (2008).

[3] P. Adamson et al., Phys. Rev. Lett. 106, 181801 (2011).

[4] Y. Ashie et al., Phys. Rev. Lett. 93, 101801 (2004); Phys. Rev. D 71, 112005 (2005).

[5] W. W. M. Allison et al., Phys. Rev. D 72, 052005 (2005).

[6] M. Ambrosio et al., Eur. Phys. J. C 36, 323 (2004).

[7] M. H. Ahn et al., Phys. Rev. D 74, 072003 (2006).

[8] B. Aharmin et al., Phys. Rev. C 72, 055502 (2005).

[9] T. Araki et al., Phys. Rev. Lett. 94, 081801 (2005).

[10] B. Pontecorvo, JETP 34, 172 (1958); V. N. Gribov and B. Pontecorvo, Phys. Lett. 28B, 493 (1969); Z. Maki, M. Nakagawa, and S. Sakata, Prog. Theor. Phys. 28, 870 (1962).

[11] See, for example, S. Weinberg, The Quantum Theory of Fields (Cambridge University Press, Cambridge, England, 1995), Vol. I.

[12] K. Nakamura et al. (Particle Data Group), J. Phys. G 37, 075021 (2010). See pp. 89 and 759.

[13] L. Wolfenstein, Phys. Rev. D 17, 2369 (1978); J. W. F. Valle, Phys. Lett. B 199, 432 (1987); M. C. GonzalezGarcia et al., Phys. Rev. Lett. 82, 3202 (1999); A. Friedland, C. Lunardini, and M. Maltoni, Phys. Rev. D 70, 111301 (2004).

[14] Recent papers have discussed the compatibility of such nonstandard interactions with a preliminary version of these MINOS data: W. A. Mann et al., Phys. Rev. D 82, 113010 (2010); J. Kopp, P. A. N. Machado, and S. J. Parke, Phys. Rev. D 82, 113002 (2010).

[15] The experiment measures an unresolved mixture of $\left|\Delta m_{31}^{2}\right|$ and $\left|\Delta m_{32}^{2}\right|$, which is referred to as $\left|\Delta m^{2}\right|$ for brevity. The parameter $\sin ^{2}(2 \theta)$ is likewise an admixture, dominated by $\theta_{23}$. Similarly for $\left|\Delta \bar{m}^{2}\right|$ and $\sin ^{2}(2 \bar{\theta})$.

[16] P. Adamson et al., Phys. Rev. D 73, 072002 (2006); 75, 092003 (2007); J. D. Chapman, Ph.D. thesis, University of Cambridge, 2007.

[17] J. J. Evans, D.Phil. thesis, University of Oxford, 2008.

[18] D. Auty, D.Phil. thesis, University of Sussex, 2010.

[19] M. C. Gonzalez-Garcia and M. Maltoni, Phys. Rep. 460, 1 (2008).

[20] K. Anderson et al., Report No. FERMILAB-DESIGN1998-01, 1998.

[21] D. G. Michael et al., Nucl. Instrum. Methods Phys. Res., Sect. A 596, 190 (2008).

[22] R. Ospanov, Ph.D. thesis, University of Texas at Austin, 2008.

[23] T. M. Cover and P. E. Hart, IEEE Trans. Inf. Theory 13, 21 (1967).

[24] J. S. Marshall, Ph.D. thesis, University of Cambridge, 2008.

[25] C. Alt et al., Eur. Phys. J. C 49, 897 (2007).

[26] G. J. Feldman and R. D. Cousins, Phys. Rev. D 57, 3873 (1998).

[27] A. I. Himmel, Ph.D. thesis, Caltech, 2011.

[28] N. E. Devenish, D.Phil. thesis, University of Sussex, 2011. 